\title{
Participação Feminina em Comitês de Programa de Simpósios da Computação
}

\author{
Ana Luiza C. Lorens, Juliana Espíndola Botelho, Ana Flávia C. Moura, \\ Bárbara D. Duarte, Mirella M. Moro
}

\author{
Universidade Federal de Minas Gerais, Belo Horizonte, Brasil \\ analorenss@ufmg.br, juliana.botelho@dcc.ufmg.br, anafciriaco@gmail.com \\ barbaradedcc.ufmg.br, mirella@dcc.ufmg.br
}

\begin{abstract}
Resumo. Este artigo segue uma metodologia diferenciada para avaliar a presença feminina nas áreas da Computação: a participação nos Comitês de Programa de simpósios da área. Nossas análises revelam a importância da interdisciplinaridade, e outras iniciativas podem se beneficiar de tais resultados.
\end{abstract}

\begin{abstract}
This paper follows an innovative characterization of Computer Science by evaluating an important part of its community: the female participation in event program committees. Our analyses reveal the importance of interdisciplinarity, and other initiatives may benefit from such results as well.
\end{abstract}

\section{Introdução}

Por razões históricas e culturais, há um desequilíbrio na participação de homens e mulheres na educação e na carreira de diversas áreas. De fato, na Academia, as diferenças são significativas na autoria de artigos [Arruda et al. 2009, Mihalcea and Welch 2015] e na colaboração internacional [Aksnes et al. 2019]. Tal falta de diversidade de gênero impacta a produtividade [Turban et al. 2019], e na Computação não é diferente. Por exemplo, estudos mostram tal impacto nas áreas de desenvolvimento de software e interação humano-computador [Burnett et al. 2015, Dray et al. 2014], e no uso amplo de software como planilhas eletrônicas [Burnett et al. 2011]. Esses estudos apontam problemas e diferenças entre os gêneros, enquanto outros discutem ações para aumentar a diversidade entre estudantes e profissionais de TI. Recentemente, paineis no maior evento científico internacional de educação em Computação discutiram as melhores práticas acadêmicas para remediar o viés de gênero na tecnologia [Garcia et al. 2020, Wolz et al. 2018].

Este artigo tem objetivo de complementar tais estudos a partir de uma metodologia diferenciada, a qual caracteriza o problema de diversidade de gênero em Computação através da participação feminina nos comitês de programa (CP) de simpósios. ${ }^{1}$ O motivo base para esse foco é: os CPs formam uma amostra mais consolidada da comunidade de uma área ou sub-área. A análise de autoria de artigos (conforme trabalhos supracitados) inclui, além de profissionais, estudantes que não necessariamente trabalharão na área do artigo após formados. De outro modo, os CPs incluem pessoas (maioria com doutorado) reconhecidas em suas áreas de atuação pela qualidade de sua pesquisa ou trabalho desenvolvido. Além disso, participantes de um CP decidem quais artigos são publicados, então possuindo presença mais decisória na comunidade.

\footnotetext{
${ }^{1} \mathrm{~A}$ metodologia foi brevemente introduzida em um pôster [Duarte et al. 2019], e aqui é expandida para considerar análises diferentes e mais amplas, bem como incluir eventos internacionais.
} 
Além de eventos brasileiros, consideramos eventos internacionais para fins de comparação. Um estudo sobre mulheres na área ao redor do mundo [Galpin 2002] aponta que a cultura própria de cada país influencia nas escolhas das mulheres. Assim, estudar eventos brasileiros é crucial para averiguar dados sobre a nossa perspectiva, com a influência da nossa cultura; mas também é relevante colocar tal perspectiva no cenário mundial, i.e., através da inclusão de eventos internacionais na nossa análise.

As questões de pesquisa que regem nosso estudo são: como é a presença feminina por evento e como a mesma evolui no tempo; como é a participação feminina nos núcleos de tais eventos; e como eventos nacionais se comparam a internacionais. Os resultados de nossas análises têm significativo potencial de utilização. Por exemplo, ao identificar as áreas com maior atuação feminina, pode-se analisar que aspectos atraem mulheres para as mesmas e enfatizá-las em ações de atração de talentos. Ademais, outras iniciativas brasileiras podem também se beneficiar dos resultados aqui apresentados. Este artigo segue com trabalhos relacionados, metodologia, avaliações e discussões sobre resultados.

\section{Trabalhos Relacionados}

Existem muitas iniciativas internacionais sobre diversidade em cursos de graduação e pós em Computação [Frieze and Quesenberry 2019, Garcia et al. 2020]. Iniciativas nacionais estão anualmente no WIT durante o Congresso da SBC. Existem ainda pesquisas sobre os motivos para mulheres escolherem a tecnologia como carreira [Duncan et al. 2020]. Mulheres são motivadas pela busca de conhecimento e flexibilidade, e homens por conhecimento e prestígio. Outro fator é gostar ou se identificar com áreas diferentes [Mihalcea and Welch 2015]. Por exemplo, um estudo com meninas em idade escolar quantifica o interesse acerca de sub-áreas [da Silva Figueiredo et al. 2017]. Outro fator frequente é a vontade de mulheres fazerem a diferença na comunidade. Por exemplo, a Inteligência Artificial resolve questões práticas que são muito atrativas, como tornar hospitais mais seguros e recuperar de desastres [Vachovsky et al. 2016].

Neste contexto, nosso objetivo é aprofundar tais questões sobre um aspecto diferenciado: a participação feminina em comitês de programa de eventos da Computação. Conforme mencionado, tal aspecto se diferencia de autoria, pois o CP é mais perene e inclui pessoas com destaque na área (ao invés de estudantes ou recém-formados). Além disso, realizamos um projeto para atrair meninas para a Computação e auxiliar as que já fizeram essa opção, ${ }^{2}$ pois conhecer experiências de outras mulheres na TI incentiva novas possibilidades na área [Martins et al. 2019]. Porém, o projeto foca em programação, e falta cobrir áreas que talvez tenham mais sucesso em atingir nossos objetivos. Assim, caracterizamos diversas áreas no contexto nacional e as comparamos com algumas internacionais, para validar resultados e ampliar potencial utilização de nosso estudo.

\section{Metodologia}

O objetivo é estudar a participação feminina em áreas de Computação através da avaliação de comitês de programa de eventos em diferentes temas da Computação. Esta seção apresenta a metodologia utilizada e lista as questões de pesquisa a serem respondidas.

Seleção de Eventos. Considera-se um conjunto representativo de eventos realizados pela

\footnotetext{
${ }^{2}$ Projeto BitGirls: http://bitgirls.dcc.ufmg.br
} 
Sociedade Brasileira de Computação ${ }^{3}$, conforme a Tabela 1. Essa tabela contém a sigla do evento, o seu nome e observações (primeiro ano de realização). Como não foram encontradas informações sobre BRACIS e WSCAD em 2011 (entre outros), os anos adjacentes foram duplicados para não excluir completamente esses eventos da análise (coluna Observações). Outros eventos brasileiros foram considerados mas não analisados por diferentes motivos: ETC (Teoria da Computação) tem apenas quatro edições; SBCM (Computação Musical) tornou-se bianual; e SBGames possui cinco trilhas com comitês separados, sendo que apenas uma é da Computação (as outras: Arte \& Design, Cultura, Educação e Indústria), razões que complicam qualquer análise quantitativa.

Como internacionais, considera-se um conjunto menor de três eventos cujas áreas são precursoras no Brasil (ICCAD, SIGMOD e SIGCOMM). Esses eventos são de excelente qualidade e realizados por sociedades científicas internacionais (ACM e IEEE). Outros eventos foram estudados, porém não incluídos por não apresentarem o CP inteiro online para maior parte do período considerado, e.g., CHI, SIGCSE E SIGGRAPH.

Tabela 1. Eventos estudados com observações de ano de fundação e coleta na terceira coluna (CPyy = Comitê de Programa do ano yy)

\begin{tabular}{lll}
\hline Evento & Simpósio Brasileiro em & $:$ Observações (ano fundação do evento) \\
\hline BRACIS & Sistemas Inteligentes & $:(2012)$ CP11 $\leftarrow$ CP12; antes era SBIA e SBRN \\
IHC & Fatores Humanos em Sistemas Computacionais & $:(1998)$ Edições bianuais até 2010 não interferiram no estudo \\
SBBD & Bancos de dados & $:(1986)$ \\
SBCCI & Circuitos Integrados e Projeto de Sistemas & $:(1983)$ junto da SBMicro; CP14 $\leftarrow$ CP13; CP19 $\leftarrow$ CP18 \\
SBES & Software: Teoria e Prática & $:(1987)$ Evento integra o CBSoft \\
SBESC & Engenharia de Sistemas de Computação & $:(2011)$ \\
SBIE & Informática na Educação & $:(1999)$ Evento integra o CBIE \\
SBMF & Métodos Formais & $:(1998)$ Evento integra do CBSoft \\
SBRC & Redes de Computadores e Sistemas Distribuídos & $:(2004)$ Não realizado em 2018, CP18 $\leftarrow$ CP19 \\
SBSC & Sistemas Colaborativos & $:(2004)$ \\
SBSeg & Segurança da Informação e Sistemas Computacionais: (2001) \\
SBSI & Sistemas de informação & $:(1988)$ CP98 $\leftarrow$ CP97, CP99 $\leftarrow$ CP00, CP19 $\leftarrow$ CP18 \\
SIBGRAPI Gráficos, Padrões e Imagens & $:(1997)$ \\
SVR & Realidade Virtual e Aumentada & $:(1998)$ \\
WebMedia Sistemas Multimídia e Web & $:(2000)$ CP11 $\leftarrow$ CP12 \\
WSCAD & Sistemas de Computação de Alto Desempenho & $:$ Observações e equiparidade com o evento brasileiro \\
\hline Evento & International Conference on & $:(1988)$ SBCCI; CP11 $\leftarrow$ CP12; CP14 $\leftarrow$ CP15 \\
\hline ICCAD & Computer Aided Design & $:(1975)$ SBBD \\
SIGMOD & Management of Data & $:(1977)$ SBRC; CP16 $\leftarrow$ CP17 \\
SIGCOMM Communications and Computer Networks &
\end{tabular}

Processamento de Dados. Para todos os eventos, o período estudado é de 2011 a 2019 - é a década mais recente, sendo mais fácil encontrar os dados necessários online, pois bibliotecas digitais contêm os artigos publicados mas nem sempre a parte inicial (foreword ou frontmatter) com os comitês. Então segue-se para a coleta dos dados realizada de maneira manual através dos websites de cada evento. Para alguns casos, foi necessário usar os anais físicos. Para cada coleta, a informação mínima é: sigla do evento, ano, e nome de participantes do CP. Também foi necessário desambiguar alguns nomes, i.e., agrupar registros diferentes para a mesma pessoa (por primeiro e último nome, e evento).

Sobre o gênero, nenhum evento oferece tal informação. Então, realizou-se uma classificação automática com uma biblioteca de processamento de linguagem natural. ${ }^{4}$

\footnotetext{
${ }^{3}$ SBC Simpósios Realizados: http://www.sbc.org.br/eventos/eventos-realizados

${ }^{4}$ Natural Language Tolkit: http://www.nltk.org
} 
Tabela 2. Tamanho do Comitê de Programa dos eventos por ano no formato (número de mulheres : total de pessoas)

\begin{tabular}{rccccccccc}
\hline evento-ano & $\mathbf{2 0 1 1}$ & $\mathbf{2 0 1 2}$ & $\mathbf{2 0 1 3}$ & $\mathbf{2 0 1 4}$ & $\mathbf{2 0 1 5}$ & $\mathbf{2 0 1 6}$ & $\mathbf{2 0 1 7}$ & $\mathbf{2 0 1 8}$ & $\mathbf{2 0 1 9}$ \\
\hline BRACIS & $43: 135$ & $43: 135$ & $35: 127$ & $48: 185$ & $23: 111$ & $40: 131$ & $49: 173$ & $50: 192$ & $45: 161$ \\
IHC & $21: 55$ & $23: 49$ & $30: 80$ & $39: 85$ & $43: 94$ & $51: 106$ & $69: 138$ & $64: 120$ & $56: 114$ \\
SBBD & $22: 52$ & $17: 43$ & $22: 59$ & $11: 44$ & $15: 48$ & $16: 50$ & $21: 52$ & $22: 51$ & $24: 64$ \\
SBCCI & $10: 90$ & $12: 108$ & $12: 106$ & $12: 106$ & $9: 79$ & $11: 86$ & $19: 139$ & $23: 143$ & $23: 143$ \\
SBES & $26: 84$ & $25: 85$ & $21: 76$ & $21: 78$ & $24: 88$ & $24: 88$ & $24: 84$ & $21: 92$ & $22: 70$ \\
SBESC & $10: 70$ & $11: 60$ & $15: 85$ & $3: 46$ & $6: 34$ & $6: 38$ & $11: 70$ & $10: 45$ & $9: 70$ \\
SBIE & $75: 153$ & $108: 217$ & $95: 195$ & $112: 222$ & $75: 149$ & $101: 226$ & $61: 124$ & $116: 247$ & $131: 313$ \\
SBMF & $8: 35$ & $8: 40$ & $8: 42$ & $8: 39$ & $9: 41$ & $9: 47$ & $10: 46$ & $11: 56$ & $5: 26$ \\
SBRC & $20: 102$ & $18: 103$ & $22: 120$ & $21: 112$ & $16: 111$ & $17: 100$ & $16: 103$ & $17: 118$ & $20: 121$ \\
SBSC & $17: 41$ & $22: 51$ & $19: 48$ & $19: 44$ & $22: 52$ & $19: 48$ & $17: 42$ & $20: 49$ & $20: 49$ \\
SBSeg & $10: 91$ & $3: 42$ & $4: 47$ & $4: 49$ & $4: 46$ & $8: 56$ & $8: 56$ & $10: 65$ & $8: 55$ \\
SBSI & $28: 69$ & $37: 98$ & $45: 108$ & $32: 83$ & $42: 105$ & $37: 94$ & $48: 128$ & $52: 131$ & $68: 175$ \\
SIBGRAPI & $23: 155$ & $8: 59$ & $6: 60$ & $33: 268$ & $14: 105$ & $22: 146$ & $23: 153$ & $20: 156$ & $20: 156$ \\
SVR & $16: 81$ & $16: 89$ & $15: 82$ & $16: 83$ & $14: 80$ & $6: 35$ & $13: 56$ & $11: 57$ & $9: 40$ \\
WebMedia & $11: 48$ & $18: 67$ & $14: 62$ & $13: 72$ & $17: 77$ & $21: 96$ & $19: 96$ & $20: 98$ & $12: 73$ \\
WSCAD & $13: 86$ & $13: 86$ & $14: 85$ & $11: 80$ & $16: 90$ & $15: 77$ & $5: 41$ & $12: 78$ & $14: 75$ \\
\hline ICCAD & $14: 117$ & $14: 117$ & $16: 103$ & $20: 105$ & $20: 105$ & $17: 112$ & $16: 124$ & $22: 119$ & $25: 130$ \\
SIGCOMM & $3: 17$ & $6: 47$ & $6: 32$ & $10: 50$ & $15: 47$ & $11: 52$ & $11: 52$ & $10: 50$ & $12: 61$ \\
SIGMOD & $38: 97$ & $23: 99$ & $20: 124$ & $17: 124$ & $30: 137$ & $28: 191$ & $30: 180$ & $31: 190$ & $15: 135$ \\
\hline
\end{tabular}

Para casos sem resultado, utilizou-se outra específica para identificar gênero. ${ }^{5}$ Os resultados de ambas foram verificados por amostragem manualmente. Após, cerca de $30 \%$ das pessoas ainda estavam com gênero não identificado (e.g., nome Ariel é ambíguo). Assim, tais nomes foram manualmente classificados com a busca das respectivas pessoas online. ${ }^{6}$

Definição do Núcleo. Para a recorrência de participação, usamos o terceiro quartil como critério; i.e., uma pessoa pertence ao núcleo de um evento se sua participação nos comitês (das edições consideradas) está entre as $25 \%$ maiores participações. Por exemplo, para o CP do evento $X$ com seis pessoas $A, B, C, D, E$ e $F$, e valores de suas participações iguais a $\{1,4,5,7,8$ e 9$\}$, respectivamente; o valor do terceiro quartil é 7,75, e as pessoas $E$ e $F$ (participações maiores que 7,75) são consideradas integrantes do núcleo de $X$.

Estatísticas Básicas. A base inicial de eventos nacionais contém 13.243 registros de participantes de CP. Após os processos supracitados, contém 4.014 registros, i.e., o número de pessoas envolvidas nos CPs. Para eventos internacionais, a base inicial possui 2.755 registros, e a final 1.205 registros de pessoas. A Tabela 2 resume o tamanho dos CPs por evento por ano no período 2011-2019, bem como o número de mulheres nos mesmos.

Ao processar os dados, notamos que muitas pessoas estão em várias edições do evento. Com eventos de tamanhos variados, esse número precisa da porcentagem para comparação uniforme. Então, propomos dois números diferentes: o de mulheres em termos de pessoas que estão no comitê, e o de participações de mulheres ao longo do tempo. Por exemplo, para o CP de $X$, a mulher $A$ participou nos anos $\left\{y_{1}, y_{2}, y_{3}\right\}$; o homem $B$ participou no ano $\left\{y_{1}\right\}$, e o homem $C$ participou nos anos $\left\{y_{2}, y_{3}\right\}$. No total de edições, o número de pessoas nesse evento é três, com percentual de $33 \%$ mulheres; já o número de participações é seis, com percentual de 50\% mulheres.

Questões de Pesquisa. A avaliação por gênero dos CPs dos eventos é guiada pelas seguintes perguntas: (1) Como a presença feminina varia de acordo com a área do evento?

\footnotetext{
${ }^{5}$ Gender Guesser: http://pypi.org/project/gender-guesser

${ }^{6}$ Temos ciência das inúmeras discussões complexas sobre sexo, gênero e identidade de gênero. Porém, tais questões estão fora do escopo deste artigo, que considera apenas nomes para obter um retrato da comunidade dividido por gênero (como vários outros estudos desse tipo).
} 
(2) Como a participação feminina evolui em cada evento? (3) Como é o núcleo dos eventos em relação a gênero? e (4) Como eventos nacionais se comparam a internacionais?

\section{Resultados Quantitativos}

Esta seção está organizada seguindo as perguntas definidas ao final da Metodologia. Uma discussão mais ampla dos resultados é apresentada na Seção 5.

\subsection{Presença Feminina por Evento Nacional}

Iniciamos as análises com uma visão global dos eventos nacionais. A Figura 1 mostra o somatório por gênero nos CPs no período 2011-2019. Como o tamanho dos CPs é muito variável (de 26 no SBMF a 313 no SBIE), os resultados estão divididos em: (a) percentual de participação, (b) número absoluto de pessoas, e (c) percentual de pessoas. Valores percentuais são importantes para saber a proporção feminina no evento, enquanto valores absolutos informam onde se encontram mais ou menos mulheres. Considerando o percentual de participação, SBIE, IHC, SBSC e SBSI se destacam por maior presença (embora SBSC possua um comitê bem menor que os demais). Por outro lado, os quatro eventos com menor proporção feminina são SBSeg, SBCCI, SIBGRAPI, SBESC e WSCAD.

Para complementar, seguindo a Metodologia, analisamos também o número de pessoas nesses eventos, visto que uma pessoa pode ter várias participações ao longo do tempo. A variação no resultado foi pequena conforme os gráficos em Figura 1(b) e (c). Para número absoluto de pessoas, destaca-se o SBSC que desce para $12^{\circ}$ lugar. O motivo é seu tamanho de comitê ser bem menor em comparação aos demais. Em contrapartida, o SIBGRAPI tem um CP grande com proporção pequena de mulheres.

\subsection{Evolução da Presença Feminina por Evento Nacional}

Esta seção analisa como a presença feminina varia ao longo do tempo. A Figura 2 ilustra o percentual de participação nos CPs com base nos números absolutos da Tabela 2. Para melhor visualização e interpretação, tal figura está dividida em: (a) eventos com maiores valores variando de $16 \%$ a $53 \%$, e (b) eventos com menores valores variando de $7 \%$ a $23 \%$ (ergo, escalas do eixo $y$ são diferentes).
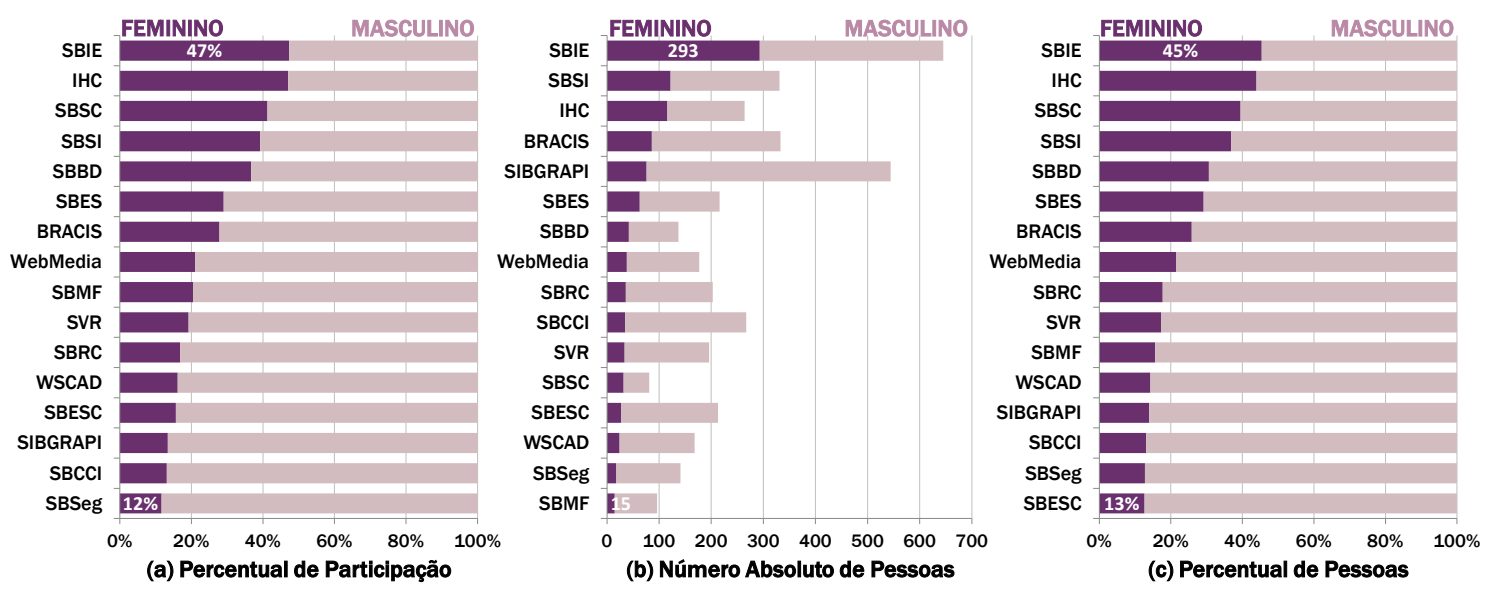

Figura 1. Presença por gênero em eventos nacionais no período 2011-2019 

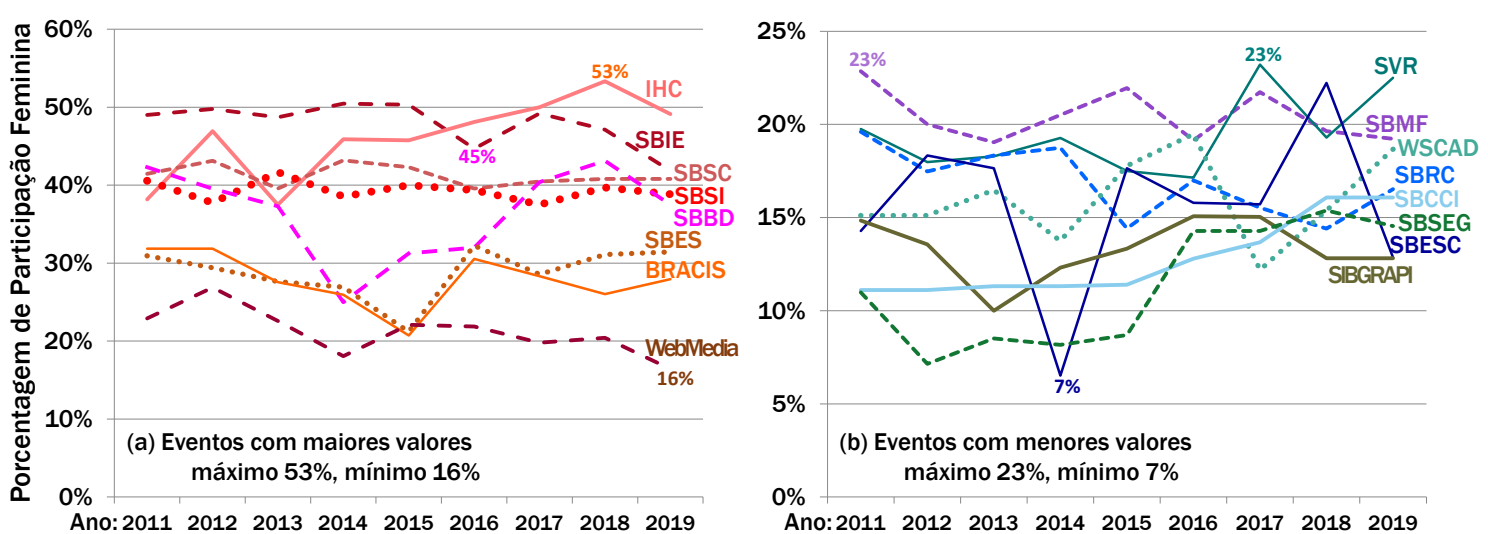

Figura 2. Evolução da porcentagem feminina nacional (eixos $y$ diferentes)

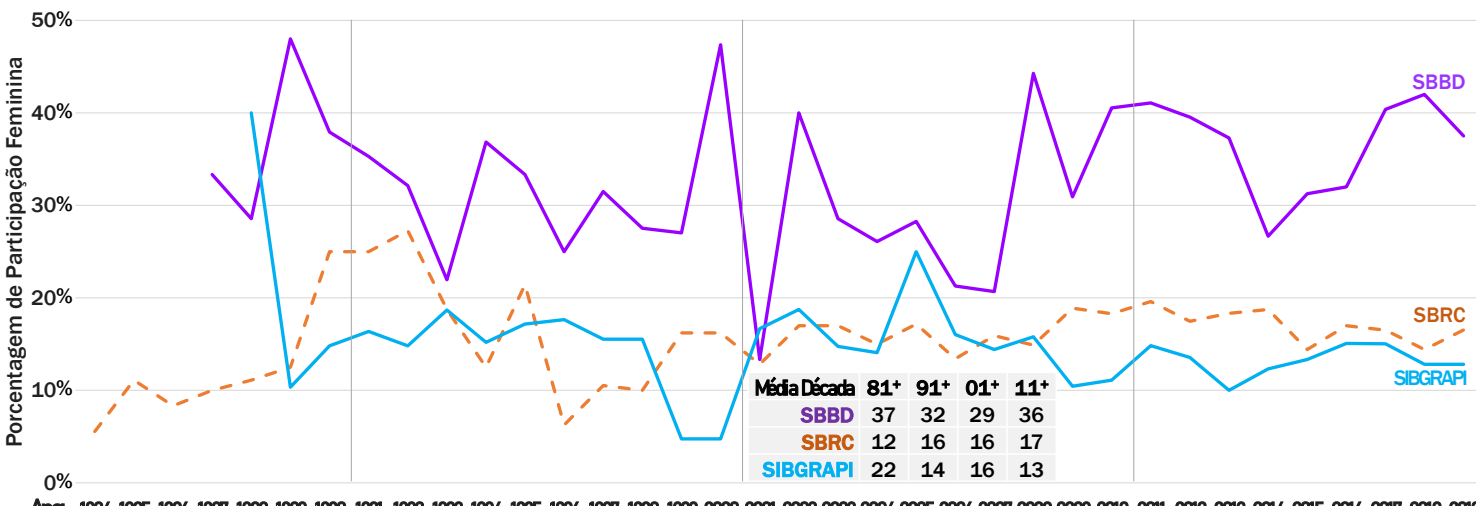

Figura 3. Evolução de quase quatro décadas, onde $81^{+}=[1981-1990]$

A Seção 4.1 mostra que a participação feminina no SBIE no total é a maior, e no SBSeg é a menor. A Figura 2(a) detalha a evolução do SBIE, cuja participação feminina fica entre $42 \%$ e $51 \%$ no período. Interessante que de 2016 em diante, o IHC apresenta a maior porcentagem feminina. Para eventos com menor participação feminina, a Figura 2(b) detalha que o SBSEG pode ter um total menor, porém a sua evolução é positiva, enquanto SIGBRAPI possui valores variando entre $10 \%$ e $15 \%$ durante a década toda.

Com SBSeg mostrando uma esperança de crescimento, realizamos uma classificação temporal, separando os dezesseis eventos em seis grupos de acordo com seu comportamento percentual: declínio para os com tendência de queda - SBMF, SBRC e WebMedia; declínio leve para os com queda mais suave que o grupo anterior - BRACIS, SBESC, SBIE e SIBGRAPI; constante para os que mantêm participação percentual uniforme - SBBD, SBSC e SBSI; aumento leve com uma projeção discreta de crescimento - SBES; aumento com projeção maior que a do grupo anterior - SBSeg, SVR e WSCAD; e aumento considerável com acréscimo de mulheres maior do que oito pontos percentuais entre 2011 a 2019 - SBCCI e IHC.

Complementando a análise temporal, avaliamos o histórico completo dos eventos de área mais antigos do Brasil ${ }^{7}$ : SBRC, SBBD e SIBGRAPI, com porcentagem de

\footnotetext{
${ }^{7}$ SECOMU e SEMISH são os eventos mais antigos realizados pela SBC, porém não são de áreas específicas como os aqui estudados.
} 

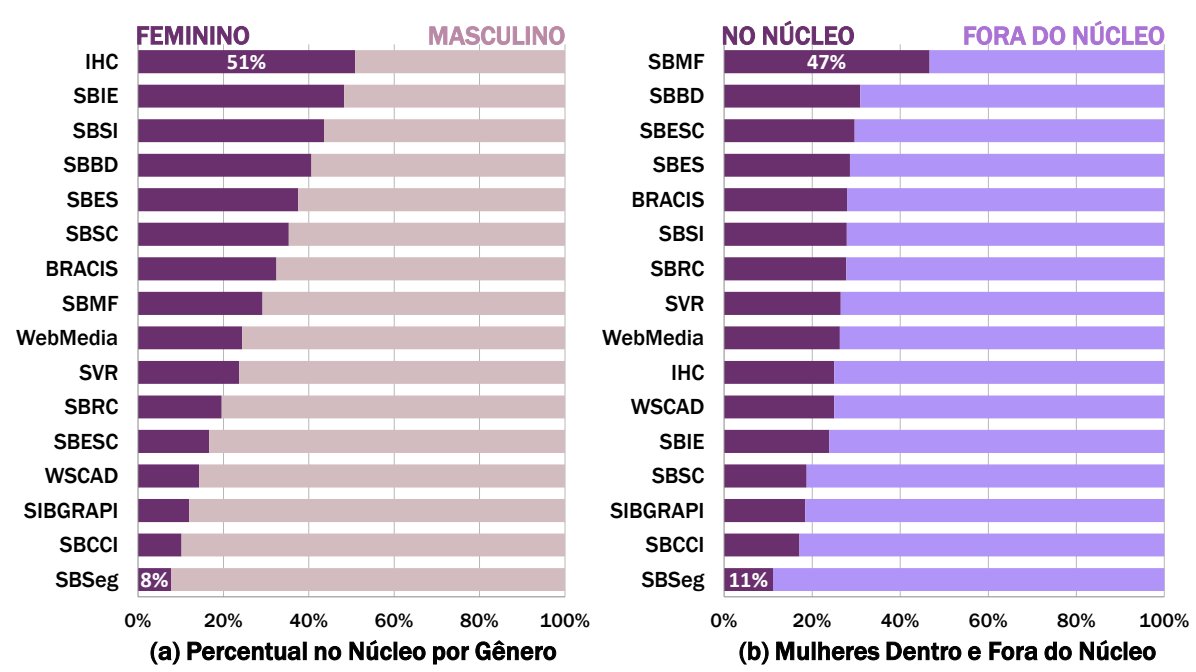

Figura 4. Presença feminina em núcleos dos eventos nacionais

participação feminina na Figura 3. Como valores flutuam, a figura contém uma pequena tabela embutida com as médias por década $\left(81^{+}\right.$é para oitenta, e assim por diante).

\subsection{Presença Feminina Mais Constante em Núcleos}

Percebemos que a participação contínua de algumas pessoas em um evento caracteriza a formação de um subgrupo mais estável dentro dos comitês. Esta seção analisa como a distribuição de gênero ocorre dentro e fora desses subgrupos, que denominamos núcleos.

A Figura 4(a) mostra a distribuição de gênero dentro dos núcleos de cada evento. Esses resultados não diferem significativamente dos resultados gerais (Figura 2), e IHC, SBIE, SBSI e SBBD mantêm-se entre os de maior proporção feminina, enquanto SBSEg, SBCCI, SIBGRAPI, WSCAD e SBESC entre os de menor proporção. A Figura 4(b) complementa essa análise ao apresentar as porcentagens de mulheres dentro e fora do núcleo por evento. Por exemplo, no IHC, das 264 pessoas que participaram, 57 estão no núcleo: 29 mulheres e 28 homens, então $51 \%$ no gráfico (a). Além dessas 29, há outras 87 mulheres fora do núcleo, que representam $75 \%$ do total de mulheres no gráfico (b).

\subsection{Análises de Eventos Internacionais}

Esta seção apresenta as análises de eventos internacionais aos pares com os respectivos nacionais de mesma área: SBBD e SIGMOD - Banco de Dados; SBRC e SIGCOMM Redes de Computadores e Sistemas Distribuídos; e SBCCI e ICCAD - Projeto de Sistemas. Aqui, é importante ressaltar que os padrões são bem parecidos com os encontrados nos eventos brasileiros, com uma diversidade de gênero pouco expressiva tanto no número de participações quanto no de pessoas. Ou seja, apesar de o problema ter suas particularidades culturais [Galpin 2002], ele é também uma questão mundial na Computação que necessita de práticas para remediar essa falta de diversidade [Wolz et al. 2018].

Nossa avaliação é dividida em três partes. Primeiro, a Figura 5(a) mostra a evolução temporal do somatório dos três CPs internacionais por gênero e ano. Apesar do número total e do número de homens aumentar, o número de mulheres nesses comitês permanece em torno de 50; ou seja, tem diminuído percentualmente. Segundo, a 

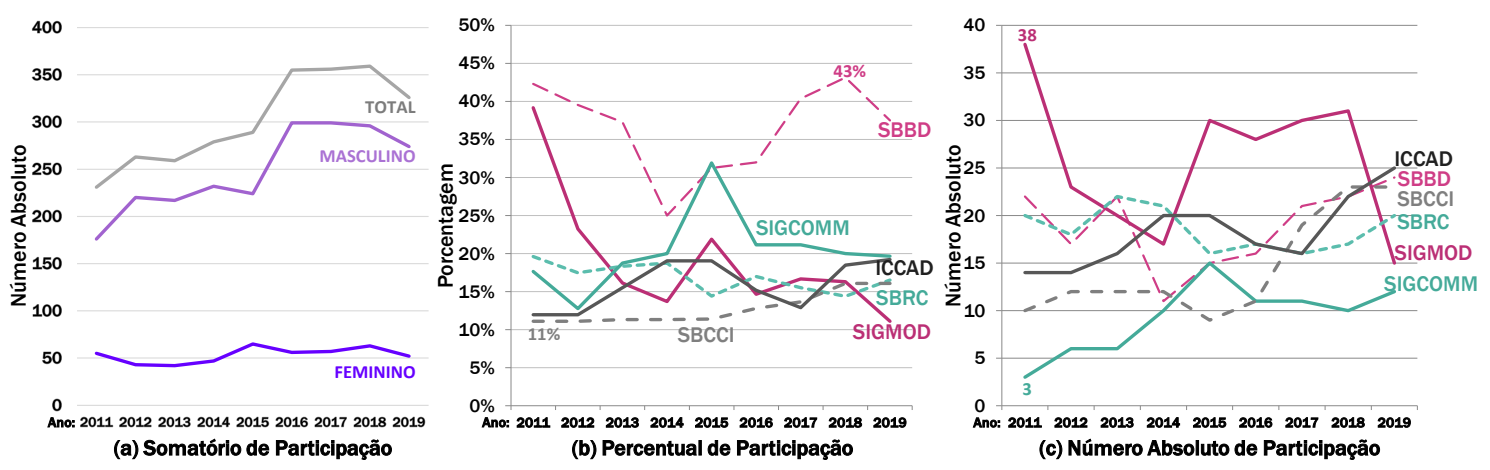

Figura 5. Evolução comparativa para eventos internacionais e nacionais

Figura 5(b) apresenta o percentual de participação feminina nos três eventos internacionais (linhas cheias) e os respectivos eventos brasileiros (linhas tracejadas de mesma cor). Interessante notar a área de banco de dados (SBBD e SIGMOD), pois enquanto o evento nacional está no topo de participação feminina, o internacional começou no topo (38\%) mas tem caído drasticamente (12\%). Essa é uma situação muito peculiar visto que, teoricamente, com a comunidade internacional inteira à disposição, é de surpreender que o número de mulheres tenha diminuído tanto. Na área de redes (SBRC e SIGCOMM), apesar da flutuação grande na participação do evento internacional, ambos mantêm tendência em torno de $13 \%$ e $20 \%$. Para projeto de sistemas (SBCCI e ICCAD), ambos mantêm-se alternando entre $11 \%$ e 19\%. Finalmente, a Figura 5(c) apresenta os valores absolutos de participação feminina para complementar os percentuais.

\section{Discussão Geral e Pontos a Enfatizar}

Esta seção discute os resultados em geral.

(1) Como a presença feminina varia de acordo com a área? Entre os eventos avaliados, os com maior presença feminina são SBIE, IHC, SBSC e SBSI, e no outro extremo estão SBESC, SBSeg, SBCCI, SIBGRAPI e WSCAD. Tais resultados corroboram as conclusões de [Arruda et al. 2009], que estuda a distribuição de publicações de brasileiros em Computação considerando as áreas do CNPq, com mais participação de autoras nas áreas de sistemas colaborativos, computadores em educação, interface humano-computador e inteligência artificial (nessa ordem). Porém, os resultados para os eventos com menos mulheres são um pouco divergentes, pois a conclusão deles inclui segurança, redes e sistemas distribuídos, e hardware e arquitetura de computadores (nessa ordem). É interessante notar que os quatro eventos mais femininos também possuem um grande viés interdisciplinar, o qual pode afetar nos resultados. Especialmente para a Informática na Educação, é notório as áreas de estudo tipicamente femininas aqui no Brasil são Educação e Ciências Sociais. ${ }^{8}$ Uma possível análise aqui seria identificar a área de atuação primária ou de formação das pessoas dos comitês de programa.

(2) Como a participação feminina evolui? O principal resultado nacional é a proporção $30 \%$ mulheres e $70 \%$ homens no total de participação ao longo do tempo, enquanto o número de mulheres matriculadas na Computação cai de $16,3 \%$ para $14 \%{ }^{9}$ no mesmo

\footnotetext{
${ }^{8}$ G1 Economia: https://g1.globo.com/economia/concursos-e-emprego/noticia/2019/09/10/mulheres-sao-maioria-nasuniversidades- brasileiras-mas-tem-mais-dificuldades-em-encontrar-emprego.ghtml

${ }^{9}$ Estatísticas INEP http://inep.gov.br/microdados
} 
período. Podemos especular que a falta de modelos na Computação não seja o maior problema, mas talvez a falta de melhor divulgação sobre a presença feminina de $30 \%$ na área? Ou não, talvez essa taxa de $30 \%$ de mulheres deva-se à interdisciplinaridade dos eventos mais femininos. Podemos também utilizar a justificativa da interdisciplinaridade a nosso favor, disseminando a informação sobre a oportunidade de trabalhar em diversas áreas de conhecimento aliadas à Computação. Avaliando os extremos, o SBIE (mais mulheres) mantém a sua taxa em torno dos $50 \%$ no período, e o SBSeg (menos mulheres) tem crescimento sutil no percentual feminino. É possível observar também o movimento de crescimento de participação feminina para outros eventos de áreas "mais masculinas" como SBSeg, SVR e WSCAD. Destaca-se também o grande aumento de mulheres no IHC, e que talvez possa o tornar "mais feminino" do grupo analisado em médio prazo.

(3) Como é a participação feminina nos núcleos dos eventos? A distribuição por gênero nos núcleos segue as tendências já apresentadas de percentual de participação.

(4) Como os pares de eventos nacionais e internacionais se relacionam? Em termos internacionais, há cerca de $18 \%$ de participação feminina. Ao sintetizar os comportamentos nacional e internacional, é visível a consonância até o ano de 2014. A partir de 2015, o par de Banco de Dados SBBD-SIGMOD apresenta uma disparidade maior. Percebe-se também que o par com a maior semelhança é o de Projeto de Sistemas SBCCI-ICCAD. Isso pode significar que áreas "mais masculinas" possuem comportamentos semelhantes mundialmente e no Brasil. Uma possível razão pode ser que, historicamente nos primórdios da Computação, homens trabalhavam mais na parte de hardware, e mulheres eram delegadas ao "trabalho menos importante" de software [Light 1999]. É possível que tal divisão tenha perdurado de alguma forma em tais áreas, visto que Redes de Computadores inclui toda a parte física da rede e Projeto de Sistemas a parte física dos circuitos.

Limites de pesquisa. Finalmente, é importante apresentar dois pontos que podem limitar nossas análises. Primeiro, existe a possibilidade de identificação errônea de gênero a partir do nome; porém, reduzimos tal limitação com uma verificação por amostragem e outra manual dos casos dúbios. Segundo, a avaliação considera o período de 2011 a 2019 pois os dados de 2010 e anos anteriores ainda estão em processo de consolidação, sendo que não conseguimos encontrar as informações de comitê de programa para todos os eventos. Mesmo assim, as análises realizadas são bem promissoras, com resultados interessantes e úteis para a identificação de aspectos que atraem mais mulheres para algumas áreas.

\section{Considerações Finais}

Este artigo conclui a primeira etapa de um projeto sobre atração de mulheres para a Computação: caracterizar as sub-áreas através da análise da presença feminina nos comitês de programa dos principais Simpósios de Computação nacionais e internacionais. $\mathrm{O}$ principal resultado é a presença maior de mulheres em áreas mais interdisciplinares, relacionadas à aplicação da computação ou com aspecto social claro, corroborando outros estudos sobre autoras mulheres [Margolis and Fisher 2003]. Também existem semelhanças no percentual feminino nos eventos nacionais e internacionais, o que confirma que há uma baixa representatividade feminina na Computação mundial [Galpin 2002], além de uma possível divulgação depreciada da participação feminina na área. O próximo passo é qualificar essa presença entrevistando as mulheres dessas áreas. Finalmente, o perfil dessas áreas pode ser melhor explorado em materiais e atividades com meninas, bem como 
servir de inspiração para outros projetos, sempre visando material de qualidade para atrair a atenção do maior número de meninas possível.

Agradecimentos. Trabalho financiado pelo Projeto CNPq Bytes \& Elas, 442265/2018-2.

\section{Referências}

Aksnes, D. W., Piro, F. N., and Rørstad, K. (2019). Gender gaps in international research collaboration: a bibliometric approach. Scientometrics, 120(2):747-774.

Arruda, D. et al. (2009). Brazilian computer science research: Gender and regional distributions. Scientometrics, 79(3):651-665.

Burnett, M. M. et al. (2011). Gender pluralism in problem-solving software. Interacting with Computers, 23(5):450-460.

Burnett, M. M. et al. (2015). SIG: gender-inclusive software: What we know about building it. In ACM CHI - Extended Abstracts, pages 857-860.

da Silva Figueiredo, K., Vitorassi, R., Monteiro, E., and de Oliveira Carneiro, S. (2017). Percepções de alunas de ensino médio sobre as subáreas da computação. In SBC WIT.

Dray, S. M. et al. (2014). Perspectives on gender and product design. In ACM CHIExtended Abstracts, pages 53-56.

Duarte, B. D., Moura, A. F. C., and Moro, M. M. (2019). Mulheres na Computação: Análises por Sub-Áreas. In SBC WIT, pages 174-178.

Duncan, A. et al. (2020). Enrollment motivations in an online graduate CS program: Trends \& gender- and age-based differences. In ACM SIGCSE, pages 1241-1247.

Frieze, C. and Quesenberry, J. L. (2019). How computer science at CMU is attracting and retaining women. Commun. ACM, 62(2):23-26.

Galpin, V. (2002). Women in computing around the world. SIGCSE Bull., 34(2):94-100.

Garcia, D. et al. (2020). Institutions share successes, failures, and advice in moving the diversity needle. In ACM SIGCSE, pages 331-332.

Light, J. S. (1999). When computer were women. Tech. and Culture, 40(3):455-483.

Margolis, J. and Fisher, A. (2003). Unlocking the Clubhouse: Women in Computing. MIT Press, Revised edition.

Martins, A., Silva, J., Santos, J., and Rebouças, A. (2019). Fatores que Atraem e Afastam as Meninas de cursos da Área de TI. In SBC WIT, pages 114-118.

Mihalcea, R. and Welch, C. (2015). What Women Want: Analyzing Research Publications to Understand Gender Preferences in Computer Science. In AAAI Workshops.

Turban, S., Wu, D., and Zhang, L. (2019). Research: When gender diversity makes firms more productive. Harvard Business Review. https://hbr.org/2019/02/ research-when-gender-diversity-makes-firms-more-productive.

Vachovsky, M. E. et al. (2016). Toward more gender diversity in CS through an artificial intelligence summer program for high school girls. In ACM SIGCSE, pages 303-308.

Wolz, U. et al. (2018). Best practices in academia to remedy gender bias in tech. In ACM SIGCSE, pages 672-673. 